\title{
Evaluation of surgically treated breast masses in children
}

\author{
Gül Doğan, Tutku Soyer, Saniye Ekinci, İbrahim Karnak, Arbay Ö. Çiftçi, Feridun C. Tanyel \\ Department of Pediatric Surgery, Hacettepe University Faculty of Medicine, Ankara, Turkey. \\ E-mail: guldemirdag_982@hotmail.com \\ Received: 9th January 2016, Revised: 16th February 2017, Accepted: 20th February 2017
}

SUMMARY: Doğan G, Soyer T, Ekinci S, Karnak İ, Çiftçi AÖ, Tanyel FC. Evaluation of surgically treated breast masses in children. Turk J Pediatr 2017; 59: 177-183.

We aimed to define the diagnostic and treatment characteristics of breast masses among female children who had undergone surgical treatment in order to figure out which children should be followed conservatively or treated surgically. We reviewed retrospectively 64 female patients operated for breast masses under the age of 18 years between 1977 and 2013. Patient demographics, symptoms, physical examination findings, size of the mass and its relation with menstrual cycle, follow-up period before and after surgery, diagnostic characteristics, laboratory analysis, diagnosis before and after surgery, indications for surgery, histopathological characteristics of the mass, postoperative complications, and recurrence rate were recorded. Histopathologic investigations among 41 girls with complete hospital records showed that 26 had fibroadenoma (FA; 63.4\%). Eleven girls among the other 23 cases with incomplete hospital records but with histopathological results have also had FA (47\%). The majority of breast masses in childhood are FA, the frequency and duration of follow up is important. If there is a mass that will not regress, that continues to grow or does not reduce in size during follow up, and for those with family history of breast cancer to definitely exclude the possibility of malignancy, surgical treatment may be appropriate.

Key words: breast masses, childhood, fibroadenoma, surgery.

Breast masses are infrequently observed during the childhood and may reflect a spectrum from simple developmental masses to inflammatory or neoplastic origins. The majority of breast masses occurring with puberty are fibroadenoma (FA). Though cystosarcoma phyllodes (CF) is a common primary breast cancer in the adolescent period, it may be observed in the prepubertal period. ${ }^{1}$ CF may show variations from a benign mass to a rapidly growing and metastasizing malignant mass. Clinical and diagnostic methods may confuse it with FA. Primary breast cancer is very rare in the childhood period with metastatic tumors especially observed in adolescent girls. ${ }^{2}$

The majority of breast masses are benign, though difficulties may be experienced during diagnosis and treatment. Additionally, there is limited information about the monitoring criteria and surgical indications for these masses. As a result, the approach may vary among pediatric surgeons. The majority of cases may be monitored very closely. During monitoring surgical treatment may be performed for cases with growing mass, those without reduction in the size of mass, in case of suspicion of malignancy, in the presence of high-risk genetic mutations or family history of breast cancer, or masses causing psychological or cosmetic problems.

Avoiding unnecessary surgical intervention has utmost importance for both preventing damage to developing breast tissue and preventing negative psychological effects. On the other hand, presence of a mass in the breast is an alarming condition for both the child and the family. Therefore, the approach and clinical follow up of to these patients remains problematic.

A retrospective clinical study was performed to define the diagnostic and treatment characteristics of breast masses among female 
children who had undergone surgical treatment to define which children should be followed conservatively or treated surgically.

\section{Materials and Methods}

After receiving permission from Hacettepe University Non-Interventional Researches Ethics Board (Decision no. GO 13/371, dated 28.06.13), the study was begun. All patients had given consent for use of clinical data for scientific purposes. Patients operated for breast masses under the age of 18 years in the Department of Pediatric Surgery between 1977 and 2013 were retrospectively evaluated.

Age, symptoms, findings at physical examination, mass size and menstrual cyclus relation, follow up before surgery, diagnostic characteristics, laboratory evaluation, diagnosis before and after surgery, indications for surgery, histopathological characteristics of the mass, postoperative complications, recurrence rate and the duration of postoperative follow up were recorded.

Ultrasonography (US) was performed. The localization and dimensions of the mass and echo characteristics were defined. In suspicion of malignancy, mammography and fine needle aspiration biopsy (FNAB) were performed.

Data was uploaded to the SPSS 16.0 program and descriptive statistics obtained. All numerical data are assessed as mean \pm standard deviation or proportions. Categorical variables (n, \%) were evaluated between the groups.

\section{Results}

There were 64 girls who underwent surgical treatment because of a breast mass. Complete medical records were available for 41 patients; histopathological evaluations alone were available for the remaining 23 patients. The mean age of patients was $14.49 \pm 2.02$ years, ranging from 8 to 18 years.

The masses were in the right breast for 20 cases $(48.8 \%)$ and in the left breast for $21(51.2 \%)$. The mean long diameter of tumors was 4.02 $\pm 2.9 \mathrm{~cm}$ with mean short diameter of 2.39 $\pm 1.8 \mathrm{~cm}$. The most common localization of masses was in the left upper quadrant (n: 12, $29 \%$ ) followed by right upper quadrant (n: $8,19.5 \%$ ), left lower quadrant (n: 7, 17\%) and behind the areola (n: 7, 17\%). The least common localization was the whole breast (n: $2,4.9 \%$ ) and the right lower quadrant (n: 1 , $2.4 \%)$.

The mean duration of complaints before admission was $12.3 \pm 2$ months, varying from 10 days to 36 months. The patients' complaints at admittance are shown in Table I.

No patient had a positive family history; 9 girls $(95.1 \%)$ were at the postpubertal period. The mass did not reveal any relation with the menstrual cycle in 36 of 39 girls (87.8\%). Three girls $(7.3 \%)$ had pain in the breast with the menstrual cycle. Two girls with breast masses $(4.9 \%)$ were prepubertal.

Evaluations included US, mammography and FNAB. While US was obtained among $87.7 \%$, mammography and FNAB were infrequently requested (Table II).

The most common preoperative diagnosis after evaluations was solid nodule (n: $23,56 \%$ ), followed by CF (n: 7, 17\%), hypoechoic lesion (n: $5,12.1 \%$ ), intraductal papilloma ( $\mathrm{n}: 2$, $4.8 \%$ ), fibroma (n: $1,2.4 \%$ ), fibrocystic disease (n: $1,2.4 \%$ ) and malignancy (n: $1,2.4 \%$ ). Preoperative diagnosis could not be obtained for 1 patient.

Table I. Complaints of Patients at Admission.

\begin{tabular}{lll}
\hline Complaints & N & $\%$ \\
\hline Mass & 25 & 61.0 \\
Breast distortion & 4 & 9.8 \\
Mass and pain & 4 & 9.8 \\
Redness and swelling & 3 & 7.3 \\
Mass and misshapen & 3 & 7.3 \\
Discharge & 2 & 4.9 \\
\hline
\end{tabular}


Table II. Methods Used for Diagnosis.

\begin{tabular}{lllll}
\hline Diagnostic method & Performed & Not performed & No data & Total \\
\hline Ultrasonography, n (\%) & $36(87.8)$ & $2(4.8)$ & $3(7.4)$ & $41(100.0)$ \\
Mammography, n (\%) & $3(7.3)$ & $35(85.3)$ & $3(7.4)$ & $41(100.0)$ \\
FNAB, n (\%) & $2(4.8)$ & $36(87.8)$ & $3(7.4)$ & $41(100.0)$ \\
\hline
\end{tabular}

FNAB: fine needle aspiration biopsy

The most common indication for surgical intervention was suspicion of malignancy (n: $26,63.4 \%$ ). This was followed by persistence of mass during follow up ( $\mathrm{n}$ : 10, 24.3\%). Increases in the sizes of masses (n: $2,4.8 \%$ ) and multiple masses (n: 2, 4.8\%) have been other indications for surgery. The indication for surgical intervention was not recorded for a patient.

For 11 cases $(27.5 \%)$, surgical treatment was planned after $7.45 \pm 6.51$ months (1-24 months) duration of follow up. The remaining 29 cases $(72.5 \%)$ had undergone surgery soon after admittance. The masses were excised under general anesthesia. Histopathologic investigations among 41 girls with hospital records showed that 26 had FA (63.4\%), 9 had fibrocystic changes (21.9\%), 2 had nodular adenosis $(4.8 \%), 2$ had tubular adenoma (4.8\%), 1 had intraductal papilloma $(2.4 \%)$, and 1 had cystosarcoma phyllodes $(2.4 \%)$.

Eleven girls among the other 23 cases with incomplete hospital records, but with histopathologic results have also had FA (47\%) (Table III).

None of the patients had postoperative complications. During a $7.12 \pm 9.8$ months follow up period, 30 cases $(75 \%)$ did not have any recurrence. However, 9 girls among $41(22.5 \%)$ had developed of a metachronous breast mass (Table IV).

\section{Discussion}

Breast masses are rare in childhood, with the majority being benign masses. According to histopathologic diagnosis these masses may be classified as FA, papilloma, CF and malignancies. The majority of these masses are FA. ${ }^{1}$ Similarly FA (63.4\%) formed the majority of diagnoses in the present study.

Primary breast cancer is very rare during childhood. Breast cancer in the childhood period comprises $0.1 \%$ of all breast cancers and less than $1 \%$ of all childhood cancers. ${ }^{2}$ A study by Tea et al. ${ }^{4}$ identified 2 primary breast cancers in a study of 43 operated patients, while in our study there was no breast cancer. Although breast cancer is rare in childhood, cases with growing mass, those without reduction in the size of mass, suspicion of malignancy in ultrasonographic evaluation and presence of high-risk genetic mutations or family history of breast cancer requires histopathologic investigations to exclude breast cancer.

The studies evaluating patients operated for breast masses in the literature have mean age

Table III. Histopathological Diagnosis of 23 Patients with Incomplete Hospital Records.

\begin{tabular}{lll}
\hline Histopathological Diagnosis & $\mathrm{n}$ & $\%$ \\
\hline Fibroadenoma & 11 & 47 \\
Fibrocystic disease & 2 & 8.6 \\
Juvenile fibroadenoma & 2 & 8.6 \\
Ductal ectasia & 2 & 8.6 \\
Chronic active inflammation & 2 & 8.6 \\
Abscess & 1 & 4.3 \\
Cystosarcoma phyllodes & 1 & 4.3 \\
Fibrocystic fibroadenomatosis & 1 & 4.3 \\
Fibroadenomatous cystic hyperplasia & 1 & 4.3 \\
\hline
\end{tabular}




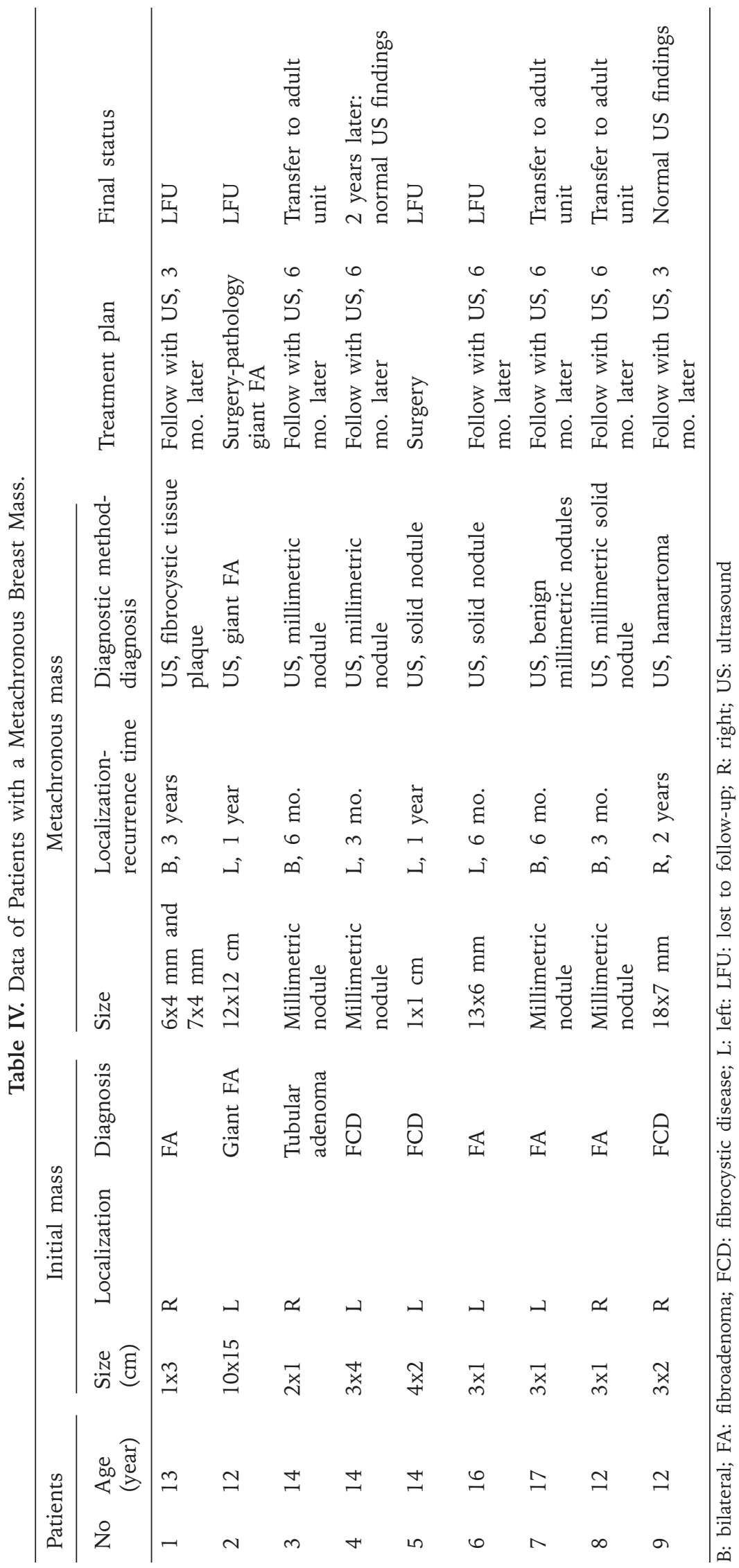


of 12,14 , and $16 . .^{3,4}$ In our study the mean age of cases was 14, and our results are similar to those in the literature.

A study by Çiftçi et al. ${ }^{3}$ on localization of masses found the upper outer quadrant was most common and reported no clear difference between right and left breast involvement. A study by Ezer et al. ${ }^{5}$ found the upper outer quadrant was most commonly involved with no difference observed between right and left breast. In another study, Gutierrez et al. ${ }^{6}$ found similar results with upper outer quadrant most common. In our study the most common localization was left upper quadrant. Twenty cases had mass in the right breast and 21 cases had mass in the left breast with no clear difference found between right and left, similar to the literature.

When masses are evaluated in terms of size, the long axis of masses in our study was mean $4 \mathrm{~cm}$, with the short axis mean $2 \mathrm{~cm}$. In other studies, the mean mass size was $4 \mathrm{~cm}, 2.6 \mathrm{~cm}$ and $3 \mathrm{~cm}$, with no clear difference compared to our study. ${ }^{3-5}$

The mean duration of complaints before admittance was 1 month in the study of Ezer et al. ${ }^{5}$; the reason for admittance has been the notification of mass in the breast. In our study the mean duration was $12.3 \pm 2$ months. While the most common reason for admittance has been the notification of mass in the breast $(61 \%)$, distortion of the breast, pain, erythema and swelling; misshapen and discharge have been other reasons. Compared to other series, the girls in the present series admitted later after the addition of other symptoms.

US along with mammography and FNAB are used for evaluation. ${ }^{3-5}$ US is very sensitive for evaluation and monitoring of fibroadenoma. ${ }^{7}$ US is radiation-free, cheap, easy to use and reliable in the differentiation of solids and cysts. In our study US was mostly used (94\%) followed by mammography (7\%) and FNAB $(5 \%)$. Due to the increase in fibroglandular tissue in proportion to fat tissue in the adolescent age group, mammography is not very beneficial. In the past, mammography was used in patients with high risk of malignancy in ultrasonographic evaluation. Currently, we prefer other radiologic modalities such as magnetic resonance imaging (MRI). It can be used to differentiate vascular lesions from normal breast tissue and to define multilobulated lesions. ${ }^{5}$ FNAB is also used for lesions suspected of malignancy thought to require close monitoring. It is very sensitive for breast masses, however as there are insufficient studies so use is limited. ${ }^{8}$

The majority of childhood breast masses are benign, as a result treatment is controversial. Some researchers suggest removal of all FAs. ${ }^{4}$ While some others use FNAB to exclude breast cancer and suggest following the patients with FA. Ezer et al. ${ }^{5}$ performed surgery on patients with growing masses or masses not reducing during follow up, those with complicated US findings, history of breast cancer in the family and with history of malignancy. Of these patients $20 \%$ were operated without following up, $80 \%$ were operated after monitoring. The mean follow-up period for these patients was 3.3 months. In our study all patients with breast masses were operated. While $72 \%$ of these patients were operated without follow up, $27 \%$ were operated after follow up. The mean duration of monitoring was 7.4 months. Of these the most common surgical intervention indication was suspected malignancy (63\%).

The study by Ezer et al. ${ }^{5}$ identified masses as $40 \%$ FA, 30\%, CF and 30\% tubular adenoma. Çiftçi et al. ${ }^{3}$ found $51 \%$ FA, $18 \%$ CF, $14 \%$ fibrocystic disease, $7 \%$ intraductal papilloma, $3 \%$ metastatic carcinoma, and $1 \%$ fat necrosis. Tea et al. ${ }^{4}$ assessed $62 \%$ FA, 9\% fibrocystic disease, $7 \% \mathrm{CF}$ and $4 \%$ ductal carcinoma. Though breast cancer is rarely observed in the pediatric age group, Gutierrez et al. ${ }^{6}$ identified $46 \%$ ductal carcinoma in their study. In our study carcinoma was not identified. In the literature FA comprise $66-76 \%$ of all breast masses, ${ }^{9,10}$ with the incidence of CF $0.3-1 \% .^{11-}$ 13 In our study similar to other studies the majority were identified as FA at $63 \%$. For the other 23 patients with no clinical and diagnostic data accessed but with pathological diagnosis, the majority of masses were identified as FA $(47 \%)$.

Çiftçi et al. ${ }^{3}$ continued postoperative follow up for 2-8 years in their study. During this time, at the end of 1 year, 1 patient operated for FA was operated again for a mass identified in the other breast, with histopathology in accordance with FA. Additionally, 1 patient had nipple collapse and 1 patient had hematoma requiring drainage. 
The mean monitoring duration in the study by Ezer et al. ${ }^{5}$ was 13 months (4-30 months) and no patient had complications or recurrence. In our study patients were monitored for an average of 7 months (1-36 months), with no patient observed to have complications. During postoperative monitoring, 9 cases $(22.5 \%)$ had metachronous masses identified in the same or in both breasts. None of these recurring/ newly identified masses were multiple. None of our patients had family history. The mean time to observation of the masses was 12 months (3-36 months).

Studies have found that in the majority of cases with surgical treatment of removal of the FA tumor, the procedure is sufficient with local recurrence in the follow up period an unusual occurrence. Tumor recurrence in the biopsy region is observed in very rare situations. Thus in situations with metachronous FA it may be considered that the original tumor was not fully removed. ${ }^{14,15}$

The etiology of FA is not clear. There are opinions that it may be linked to deviations of normal development and involution and abnormal growth of stromal cells, or is a hyperplastic nodule or is due to lobular stimulation of estrogen and excessive response of estrogen. The varying opinions related to the etiology lead to the consideration that FA does not have a singular structure, indicating varying characteristics with different diseases. This situation includes the possibility that clinical progression may be linked to each different characteristic. As a result, it should be noted that the disease may regress on its own.

If FA is a deviation of normal development and involution or abnormal growth of stromal cells, surgical treatment may be appropriate. If it is a hyperplastic nodule, instead of immediate surgery, follow up may be more appropriate. Another finding supporting follow up is the $10-15 \%$ possibility that FA will disappear without treatment. For example, in eccrine syringofibroadenoma the malignancy risk is low, and there is a possibility of regression. ${ }^{16}$ Just as the follow up duration is important, the duration to regression may be important. The malignancy risk below the age of 25 is low; however due to the possibility of regression, it is recommended to wait several menstrual cycles before surgical excision. ${ }^{17}$ In conclusion, it is not clear what the progression of disease will be in that patient due to clinical and diagnostic characteristics. When the low malignancy risk and possibility of regression are considered, follow up the mass for a few months, and later performing surgical excision if the mass does not disappear may be appropriate.

The developmental risk of carcinoma from FA sourced in epithelial elements is stated as $0.1-0.3 \% .^{3}$ When patients are followed up, this possibility should be noted. Additionally, in patients with giant FA and juvenile FA the treatment considered should be surgery without follow up because giant FA may be confused with CF. Juvenile FA may cause strain and ulceration in the breast due to rapid growth. It may have clear hyperplasia in the epithelium, and sometimes is atypical at the boundaries. As a result, long-term follow up after surgery is necessary.

The treatment for a benign mass is surgery; follow-up is not a form of treatment. Additionally, the psychological effects on a patient with breast mass must be considered. It should be explained to the family that it may cause cosmetic problems and regional pain. However, in spite of this families may choose surgery due to the conservative approach causing more anxiety. As a result, the frequency and duration of follow up is important. If there is a mass that will not regress, there is no benefit to long term follow up and delaying surgical treatment. For FA that continue to grow or do not reduce in size during follow up, and for those with family history of breast cancer to definitely exclude the possibility of malignancy, removal may be appropriate.

\section{REFERENCES}

1. Simmons PS. Diagnostic considerations in breast disorders of children and adolescents. Obstet Gynecol Clin North Am 1992; 19: 91-102.

2. Bond SJ, Buchino JJ, Nagaraj HS, Mc Masters KM. Sentinel lymph node biopsy in juvenile secretory carcinoma. J Pediatr Surg 2004; 39: 120-121.

3. Çiftçi AÖ, Tanyel FC, Büyükpamukçu N, Hiçsönmez A. Female breast masses during childhood: A 25-year review. Eur J Pediatr Surg 1998; 8: 67-70.

4. Tea MK, Asseryanis E, Kroiss R, Kubista E, Wagner T. Surgical breast lesions in adolescent females. Pediatr Surg Int 2009; 25: 73-75. 
5. Ezer SS, Oguzkurt P, Ince E, Temiz A, Bolat FA, Hicsonmez A. Surgical treatment of the solid breast masses in female adolescents. J Pediatr Adolesc Gynecol 2013; 26: 31-35.

6. Gutierrez JC, Housri N, Koniaris LG, Fischer AC, Sola JE. Malignant breast cancer in children. J Surg Res 2008; 147:182-188

7. Harper AP, Kelly FE, Noe JS. Ultrasound breast imagingthe method of choice for examining the young patient. Ultrasound Med Biol 1981; 7: 231-237.

8. Wakely PE Jr, Powers CN, Frable WJ. Metachronous soft-tissue masses in children and young adults with cancer: correlation of histology and aspiration cystology. Hum Pathol 1990; 21: 669-677.

9. Farrow JH, Ashikari H. Breast lesions in young girls. Surg Clin North Am 1969; 49:261- 269.

10. Turbey WJ, Buntain WL, Dudgeon DL. The surgical management of pediatric breast masses. Pediatrics 1975; 56:736-739.

11. West KW, Rescorla FJ, Scherer LR 3rd, Grosfeld JL. Diagnosis and treatment of symptomatic breast masses in the pediatric population. J Pediatr Surg 1995; 30:186-187.
12. Kabukçuoğlu F, Güngör N, Evren İ. Adolesan yașta filloides tümör (3 olgu). Türkiye Ekopatoloji Dergisi 1998; 4: 126-129.

13. Rajan PB, Cranor ML, Rosen PP. Cystosarcoma phyllodes in adolescent girls, and young women: A study of 45 patients. Am J Surg Pathol 1998; 22: 64-69.

14. Iglehart D. The breast. In: Sabiston D (ed). Sabiston Textbook of Surgery. Philadelphia: Saunders, 1997: 566-567.

15. Malt R. Benign condition of breast. In: Wood CW, Morris P (ed). Oxford Textbook of Surgery (2nd ed), Vol. 1. New York: Oxford University, 1994: 796-799.

16. Tey HL. Characterizing the nature of ecrine syringofibroadenoma: Illustration with a case showing spontaneous involution. Clin Exp Dermatol 2009; 34 e66-e68.

17. Deschenes L, Jacob S, Fabia J, Christen A. Beware of breast fibroadenomas in middle-aged women. Can J Surg 1985; 28: 372-374. 